\title{
Laser-to-Laser Remote Transfer and Synchronization with Sub-fs Precision over a 3.5 km Fiber Link
}

\author{
$\underline{\text { Kemal Safak }}{ }^{1,2,}$, Ming Xin ${ }^{1}$, Michael Y. Peng ${ }^{3}$, Patrick T. Callahan ${ }^{3}$, and Franz X. Kärtner ${ }^{1,2,3,4}$ \\ ${ }^{1}$ Center for Free-Electron Laser Science, Deutsches Elektronen-Synchrotron, Notkestrasse 85, Hamburg 22607, Germany \\ ${ }^{2}$ Physics Department, University of Hamburg, Luruper Chaussee 149, 22761 Hamburg, Germany \\ ${ }^{3}$ Department of Electrical Engineering and Computer Science and Research Laboratory of Electronics, \\ Massachusetts Institute of Technology Cambridge, Massachusetts 02139, USA \\ ${ }^{4}$ The Hamburg Center for Ultrafast Imaging, Luruper Chaussee 149, 22761 Hamburg, Germany \\ *E-mail: kemal.ahmed@cfel.de
}

\begin{abstract}
We present recent progress made in optical, highprecision, long-term stable timing distribution systems for synchronization of next generation $X$-ray free-electron lasers. Timing jitter characterization of the optical master oscillator shows less than 170 as integrated jitter for frequencies above 10 $\mathrm{kHz}$, limited by the detection noise floor. Timing stabilization of a 3.5-km polarization maintaining fiber link is successfully achieved with an unprecedented RMS drift of 0.18 fs over 80 hours of operation using balanced optical cross-correlation detection scheme. The output of the fiber link is used to remotely synchronize a second mode-locked laser, and continuous operation over 40 hours shows only 0.88 fs RMS drift between the two lasers under typical facility conditions.
\end{abstract}

Keywords - timing; timing jitter; noise characterization; frequency transfer; timing transfer; optical correlators; optical fiber applications

\section{INTRODUCTION}

Modern large-scale scientific facilities such as X-ray freeelectron lasers (FELs) require extremely precise timing distribution systems to synchronize radio frequency and optical sources across distances ranging from hundreds of meters to several kilometers $(\mathrm{km})$, in order to generate short $\mathrm{X}$-ray pulses on the order of a few femtoseconds (fs) [1]. FELs currently in their design stage are predicted to be able to generate sub-fs X-ray pulses, and the European XFEL, the largest FEL currently under construction, will have a length of $3.5-\mathrm{km}$. Therefore, there is a need for timing distribution and synchronization systems delivering sub-fs precision over multi-km distances, which has not been achieved so far.

Over the past decade, we have developed a pulsed-optical timing distribution system which uses ultralow-noise pulse trains generated from a mode-locked laser referenced to a microwave standard as its timing signal [2]. Then, this ultralow-noise signal is transferred through timing-stabilized fiber links to the end stations, where efficient and robust synchronization is realized using balanced optical crosscorrelation (BOC) detection scheme [3]. This paper reports a complete set of results corresponding to a laser-to-laser remote synchronization experiment over a $3.5-\mathrm{km}$ timing-stabilized fiber link. First, we show the timing jitter characterization results of the optical master oscillator (OMO). Then, the

The authors acknowledge financial support by the Center for FreeElectron Laser Science (CFEL) at Deutsches Elektronen-Synchrotron (DESY), Hamburg, a research center of the Helmholtz Association, Germany. The authors are indebted to John M. Fini, Eric Monberg, Lars Grüner Nielsen, and Man Yan from OFS Fitel for many valuable discussions concerning the $3.5 \mathrm{~km}$ PM link. stabilization of our $3.5-\mathrm{km}$ long fiber link is presented and finally, successful long-term remote synchronization of another mode-locked laser to the OMO via this link is detailed.

\section{ATTOSECOND LASER TIMING JITTER CHARACTERIZATION}

The low-noise performance of the OMO, being the heart of the timing distribution scheme described in this paper, is of tremendous importance, especially for frequencies higher than the locking bandwidth of the feedback loops employed. Because of their ultralow noise, which is confirmed experimentally to be in the attosecond (as) range [4], it has been anticipated that mode-locked lasers could synchronize large-scale scientific facilities such as X-ray FELs requiring extremely high timing accuracy potentially with sub-fs precision [2]. Hence, timing jitter characterization of modelocked lasers is a crucial step for precise timing distribution systems, in order to choose the laser with lowest possible noise. However, the conventional jitter measurement method using high-speed photodetectors and mixers usually cannot characterize jitter below $1 \mathrm{fs}$, due to the excess phase noise in the photodetection process and due to the limited timing resolution of the mixer. Recently, jitter measurements using the BOC technique, which can extract the timing information much more precisely than microwave techniques, have been demonstrated with attosecond jitter resolution [5].

\section{A. Experimental Setup}

We characterized the jitter performance of the OMO used in the present study, Origami-15 from Onefive $\mathrm{GmbH}$, with the BOC method as illustrated in Fig. 1. The BOC scheme used here is polarization-dependent type-II second-harmonic generation in a periodically-poled potassium titanyl phosphate (PPKTP) crystal. The OMO was a mode-locked laser operating at $1554 \mathrm{~nm}$ center wavelength with $+22.4 \mathrm{dBm}$ average output power, $150 \mathrm{fs}$ pulse width and $216.66 \mathrm{MHz}$ repetition rate. The slave laser was another Origami-15 with slightly different output parameters. It had a central wavelength at $1553 \mathrm{~nm}$, a repetition rate of $216.67 \mathrm{MHz}$ with an output power of +22.1 $\mathrm{dBm}$ and a pulse duration of $172 \mathrm{fs}$. 


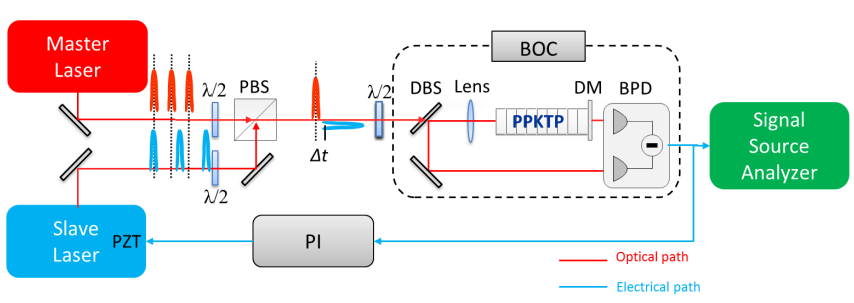

Fig. 1: Experimental setup for timing jitter characterization using a BOC (the main elements of a BOC are shown within the dashed box). Abbreviations: $\lambda / 2$ : half-wave plate; PBS: polarization beam splitter; $\Delta \mathrm{t}$ : timing mismatch between two pulse streams; DBS: dichroic beam splitter; PPKTP: periodically-poled potassium titanyl phosphate crystal; DM: dichroic mirror; BPD: balanced photodetector; PI: proportional-integral servo controller; PZT: piezoelectric transducer.

Starting with these two lasers, we combined their pulse streams in a BOC. The error signal was fed back to the piezoelectric transducer (PZT) of the slave laser, in order to lock its repetition rate to that of the master laser. Once locked, the laser timing jitter could be observed directly for frequencies beyond the locking bandwidth. Since the two lasers are almost identical, the measured spectral power density can be divided by two to find the jitter contribution of one laser. Finally, the power spectral density is converted to jitter spectral density by using the timing sensitivity of the BOC which was measured to be $0.36 \mathrm{mV} / \mathrm{fs}$ with a balanced photodetector (BPD) of $2 \times 10^{3}$ V/A transimpedance gain and $1-\mathrm{MHz}$ bandwidth in this experiment. From a simple feedback-loop analysis, the locking bandwidth of the BOC can be calculated as [6]:

$$
f_{\text {Locking }}=\sqrt{\frac{K}{2}\left[K-2 f_{P I}+\sqrt{\left(\mathrm{K}-2 f_{P I}\right)^{2}+4 f_{P I}^{2}}\right]}
$$

where $K=K_{B O C} K_{P I} K_{P Z T} /\left(2 \pi f_{\text {rep }}\right)$ and $K_{B O C}(\mathrm{~V} / \mathrm{fs})$ and $K_{P Z T}$ $(\mathrm{Hz} / \mathrm{V})$ are the sensitivities of the BOC and of the slave laser PZT, respectively. $K_{P I}$ and $f_{P I}$ are the gain and corner frequency of the PI servo controller, and $f_{\text {rep }}$ is the repetition rate of the master laser. By using some typical values observed in our experiment: $K_{\mathrm{BOC}}=1 \mathrm{mV} / \mathrm{fs}, K_{P Z T}=10 \mathrm{~Hz} / \mathrm{V}, f_{\text {rep }}=216 \mathrm{MHz}$, $K_{P I}=1, f_{P I}=1 \mathrm{kHz}, f_{\text {locking }}$ can be calculated to be approximately $6 \mathrm{kHz}$.

\section{B. Results \& Discussion}

Fig. 2(a) shows the jitter measurement results of the OMO for varying servo controller gain settings over the frequency range from $10 \mathrm{~Hz}$ up to $1 \mathrm{MHz}$. As can be inferred from Eq. (1), a high servo gain value would lead to a large locking bandwidth, which in turn would not allow the inherent jitter of the laser to be observed for low frequencies. Therefore, the measured timing spectral densities with gain values above 10 $\mathrm{dB}$ on Fig. 2(a) correlate only with the other spectra for frequencies higher than $10 \mathrm{kHz}$. As the servo gain is decreased, the locking bandwidth is reduced and the inherent jitter of the laser is revealed for offset frequencies below $1 \mathrm{kHz}$ (see the spectra for $0 \mathrm{~dB}$ gain or lower on Fig. 2(a)). Due to the low timing jitter of the laser, the jitter spectral density above 30
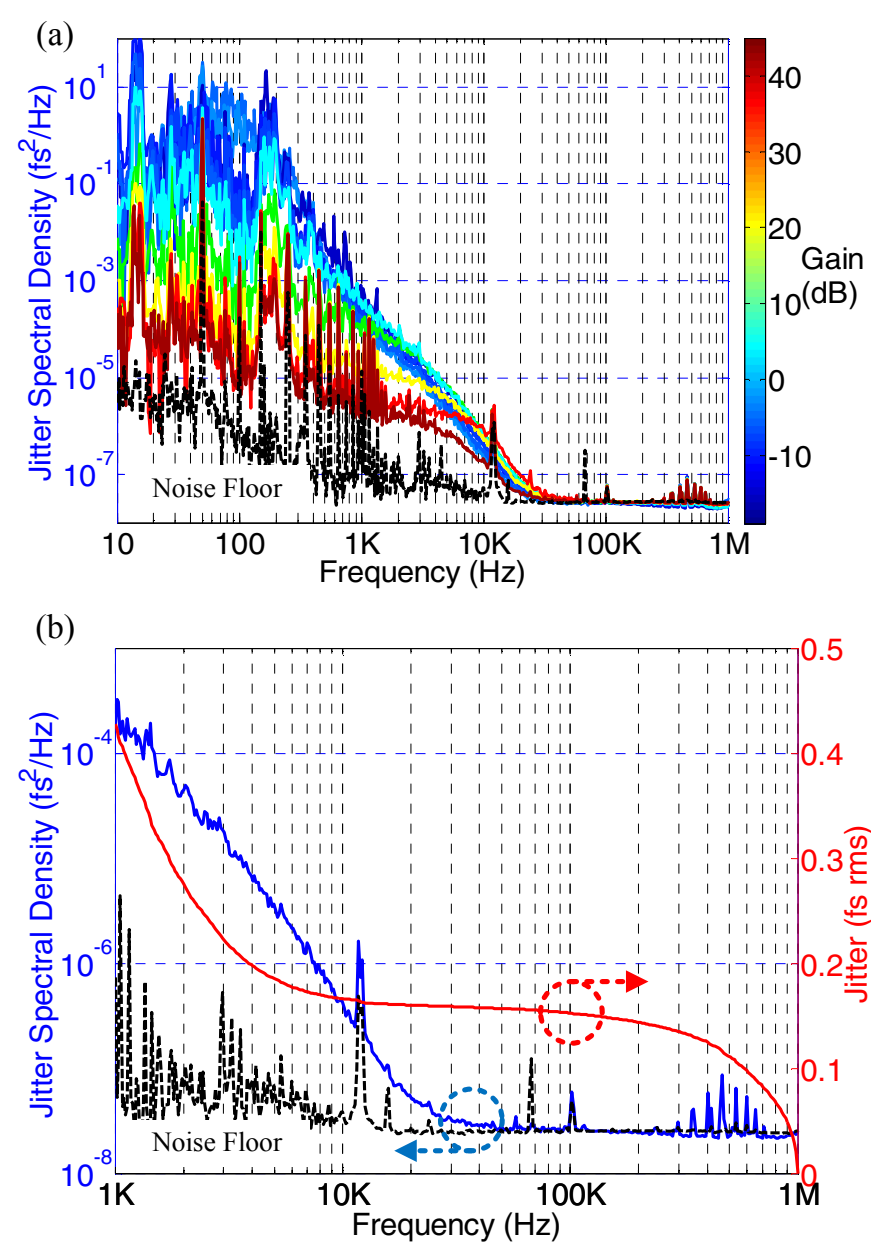

Fig. 2: (a) Timing jitter spectral density of the OMO for varying servo controller gain. The color code indicates the gain values of the individual spectra. (b) Jitter spectrum for $0 \mathrm{~dB}$ servo gain (blue curve) and its integrated timing jitter (red curve). The noise floor is determined by measuring the output of the balanced detector when the input is blocked and plotted in dashed-black on both figures.

$\mathrm{kHz}$ is obscured by the noise floor of the measurement (black dashed curve on Fig. 2(a) and 2(b)).

The measurement result shown in Fig. 2(b) corresponds to the timing jitter spectral density between $1 \mathrm{kHz}$ and $1 \mathrm{MHz}$ taken for $0 \mathrm{~dB}$ servo gain. The integration of the spectrum results in an extremely low timing jitter of approximately 400 as, for frequencies between $1 \mathrm{kHz}$ and $1 \mathrm{MHz}$. Due to the fact that operational locking bandwidths for our applications are expected to be higher than $10 \mathrm{kHz}$, the relevant frequency range for the integrated jitter is between $10 \mathrm{kHz}$ and $1 \mathrm{MHz}$. In this range, the OMO exhibits an integrated jitter of 170 as, although since the laser jitter above $30 \mathrm{kHz}$ is already limited by the detection noise floor, the true value will be even lower. Therefore this laser is well suited for the development of a precise timing distribution system. 


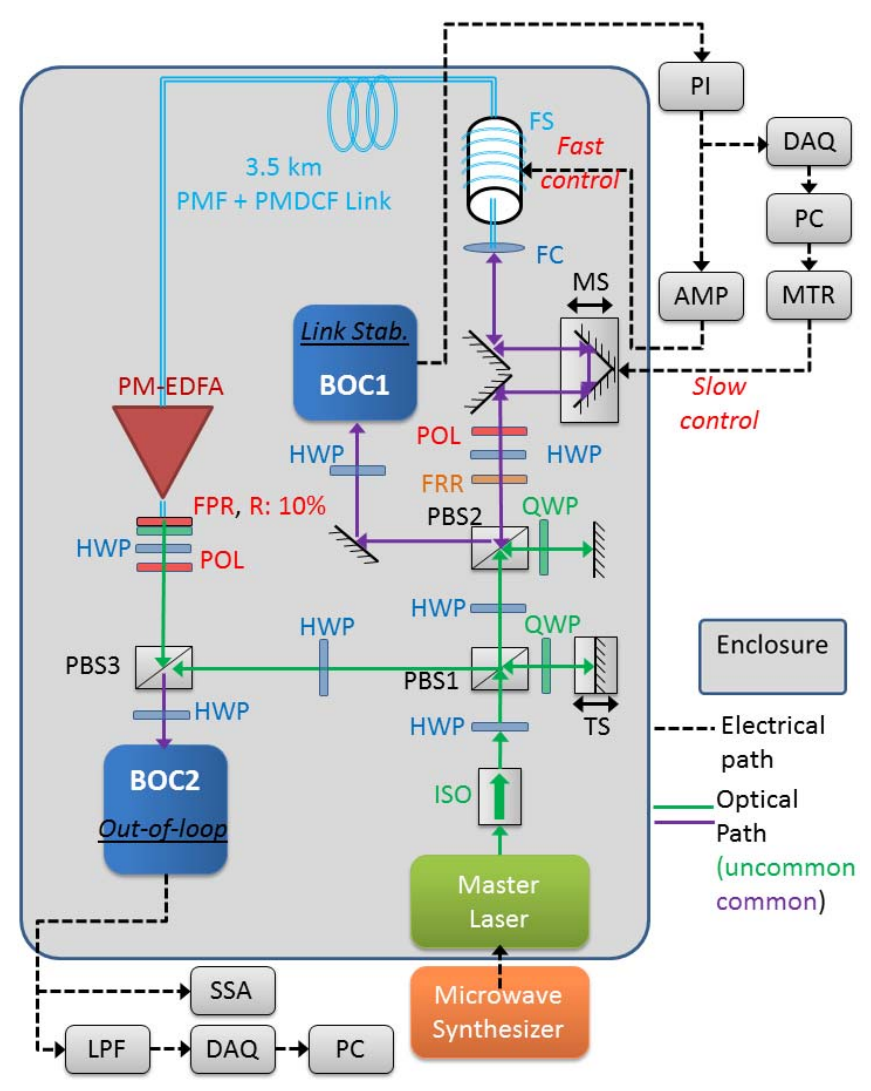

Fig. 3: Schematic of the link-stabilization experiment. Abbreviations: ISO: isolator; HWP: half-wave plate; QWP: quarter-wave plate; PBS: polarization beam splitter; TS: translation stage; FRR: Faraday rotator; POL: polarizer; MS: motorized stage; FC: fiber coupler; FS: fiber stretcher; PMF: PM fiber; PMDCF: PM dispersion compensated fiber; PI: proportional-integral servo controller; SSA: signal source analyzer; LPF: low pass filter; DAQ: data acquisition card; AMP: amplifier; PC: computer; MTR: motor controller; PM-EDFA: PM Er doped fiber amplifier; FPR: fiber partial reflector; BOC: balanced optical cross-correlator.

\section{TIMING StABILIZATION OF A 3.5-KM FiBER LINK}

Drift- and jitter-free distribution of a pulsed optical signal is crucial for the construction of a high precision timing system, in order to preserve the low noise properties of the OMO pulses during the delivery to remote locations. In an unstabilized link, environmental fluctuations (such as temperature, humidity, mechanical stress etc.) will induce errors in the output pulse arrival times. In order to stabilize the time of flight of the pulses in the fiber link to the sub-fs regime, first the round-trip timing error must be determined with high precision. Here, we employ a BOC to detect the time of flight fluctuations in the fiber, and adjust a variable delay line within the link to compensate for the detected error.

\section{A. Experimental Setup}

The experimental setup for link-stabilization is shown in Fig. 3. The OMO repetition rate was locked to a microwave synthesizer with a $10-\mathrm{Hz}$ bandwidth in order to avoid long term frequency drifts. The pulse train emitted from the OMO was coupled into a $3.5-\mathrm{km}$ long, polarization-maintaining
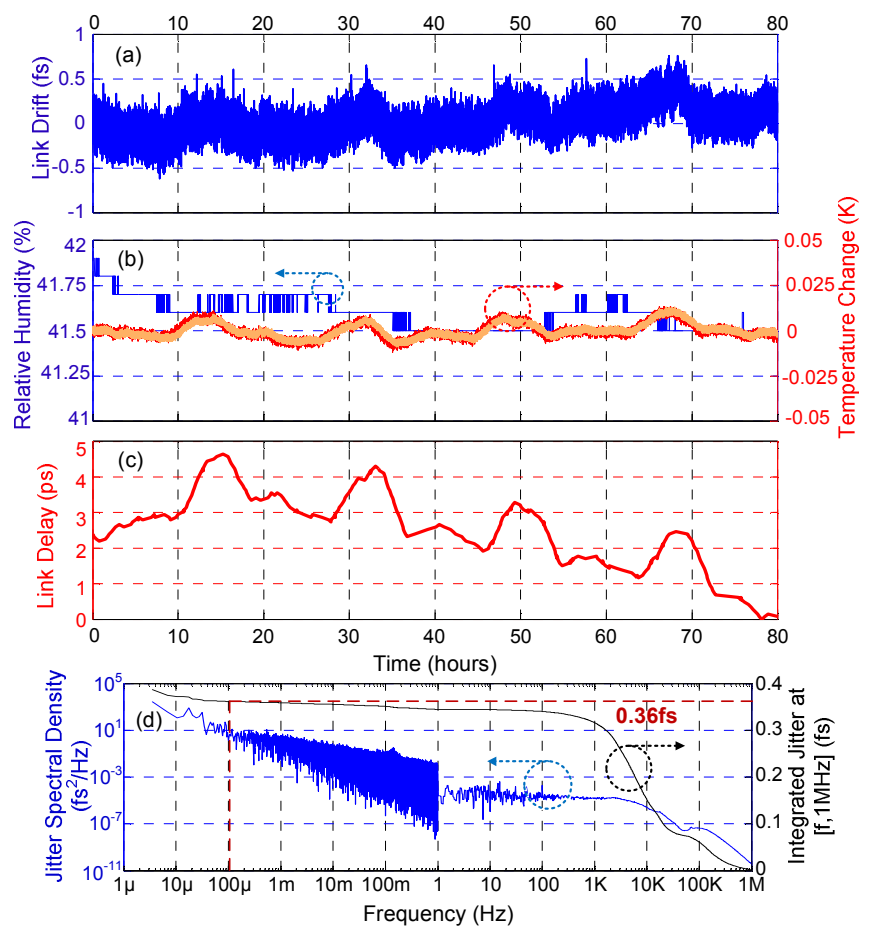

Fig. 4: Link-stabilization experiment results. (a) Timing drift for 80 hours of operation measured by BOC2; (b) environmental conditions during the measurement, blue curve shows the humidity change around the fiber link whereas red and orange curves show the temperatures on the case containing the fiber spool and on the breadboard respectively; (c) corrected link delay by the motorized stage; (d) blue curve: timing jitter spectral density between 4 $\mu \mathrm{Hz}$ and $1 \mathrm{MHz}$, black curve: its integrated jitter.

(PM) fiber link whose length was chosen to match that of the European XFEL. The link was dispersion-compensated up to the $3^{\text {rd }}$ order and was spooled inside an aluminum case. PM fiber was chosen over standard single mode (SM) fiber, as previous results obtained with SM fiber showed substantial polarization-mode-dispersion effects in the out-of-loop linkstabilization measurements [7].

In the last section of the timing link there was a custom built, bi-directional PM-EDFA to boost the output power of the link. The input power to the link at the fiber collimator (FC on Fig. 3) was $+8 \mathrm{dBm}$ and the overall one-way link loss was $-8 \mathrm{~dB}$, resulting in only $+0 \mathrm{dBm}$ input power to the PM-EDFA in the forward direction. The EDFA was operated with a pump current of $850 \mathrm{~mA}$ and the output power from the link was $+13 \mathrm{dBm}$. $+3 \mathrm{dBm}$ average power was reflected back by the fiber partial reflector (FPR with $10 \%$ back reflection on Fig. 3), and was reamplified by the EDFA to $+13 \mathrm{dBm}$, which was close to the onset of fiber nonlinearities.

The back-propagated pulse was then combined with a new laser pulse at PBS2 in the orthogonal polarization. BOC1, the in-loop detector, measured the relative timing error between these pulses and generated an error voltage. The error signal was then applied to a fiber stretcher as a fast control and to a motorized stage for slow control over an extended range of 30 $\mathrm{mm}$. For $+16 \mathrm{dBm}$ average power from the reference port and $+2 \mathrm{dBm}$ average power returning from the link, the timing sensitivity of the in-loop BOC was $14 \mathrm{mV} / \mathrm{fs}$ at a BPD 
transimpedance gain of $2 \times 10^{6} \mathrm{~V} / \mathrm{A}$ and a bandwidth of 150 $\mathrm{kHz}$. BOC2 served as the out-of-loop detector to detect the timing drifts between the traveled pulses and the newly emitted ones. Average input powers were $+11 \mathrm{dBm}$ and +19 $\mathrm{dBm}$ for the link output and reference power respectively, and the achieved sensitivity was $275 \mathrm{mV} / \mathrm{fs}$ using the same BPD settings as in $\mathrm{BOCl}$. To measure the low-frequency jitter, the out-of-loop BOC voltage was first filtered by a $1-\mathrm{Hz}$ low pass filter (LPF) to avoid aliasing, and then recorded by a DAQ card with a $2-\mathrm{Hz}$ sampling rate.

In order to minimize the drifts coming from length fluctuations in the out-of-loop reference paths (mainly caused by temperature changes), all elements of the setup were mounted on a temperature-stabilized breadboard and shielded with humidity and acoustic insulation material.

\section{B. Results \& Discussion}

Timing stability of the $3.5-\mathrm{km}$ long PM fiber link was continuously monitored for 80 hours without interruption. Fig. 4(a) shows the out-of-loop timing drift results of the linkstabilization experiment. The remaining drift was only $0.18 \mathrm{fs}$ RMS and 1.4 fs peak-to-peak, which is a record-low value for fiber transmission over such a long distance. During the course of the measurement, the humidity (blue curve on Fig. 4(b)) was stabilized to within $0.3 \%$ inside the enclosure, with the help of the humidity insulation. The maximum deviation of the temperature on the case of the fiber spool was about $0.025 \mathrm{~K}$ and showed similar fluctuations as the temperature of the breadboard since it was kept inside the enclosure. In the meantime, the motorized stage compensated for 5 ps change in the link length. The output of BOC2 (on Fig. 3) was also sent to a signal source analyzer (SSA) to measure the power spectral density of the residual timing jitter above $1 \mathrm{~Hz}$. Then by combining with the Fourier-transformed timing drift over 80 hours, we obtained the frequency spectrum of the timing jitter between $4 \mu \mathrm{Hz}$ and $1 \mathrm{MHz}$ (see Fig. 4(d)). The calculated integrated jitter between $100 \mu \mathrm{Hz}$ and $1 \mathrm{MHz}$ was only $0.36 \mathrm{fs}$.

\section{REMOTE LASER-TO-LASER SYNCHRONIZATION}

Although timing-stabilization of the fiber link assures the transmission of low jitter properties of the OMO output pulses, effective synchronization of the lasers (i.e. pump/probe laser) at the end stations determines the real output of a timing distribution system, as it directly affects the timing resolution of the experiment which is to be performed. For the first time in this paper, we show a successful remote synchronization of a second mode-locked laser to the OMO over a distance of 3.5 $\mathrm{km}$ in a laboratory environment.

\section{A. Experimental Setup}

Fig. 5 shows the schematic of the remote laser-to-laser synchronization setup. Here, the link stabilization part from Fig. 3 remained unchanged except that the fiber spool was taken out of the enclosure. Instead, the spool was placed on the optical table in the laboratory and exposed to the environmental fluctuations. In this way, we aimed to create more realistic operational conditions for the fiber link and

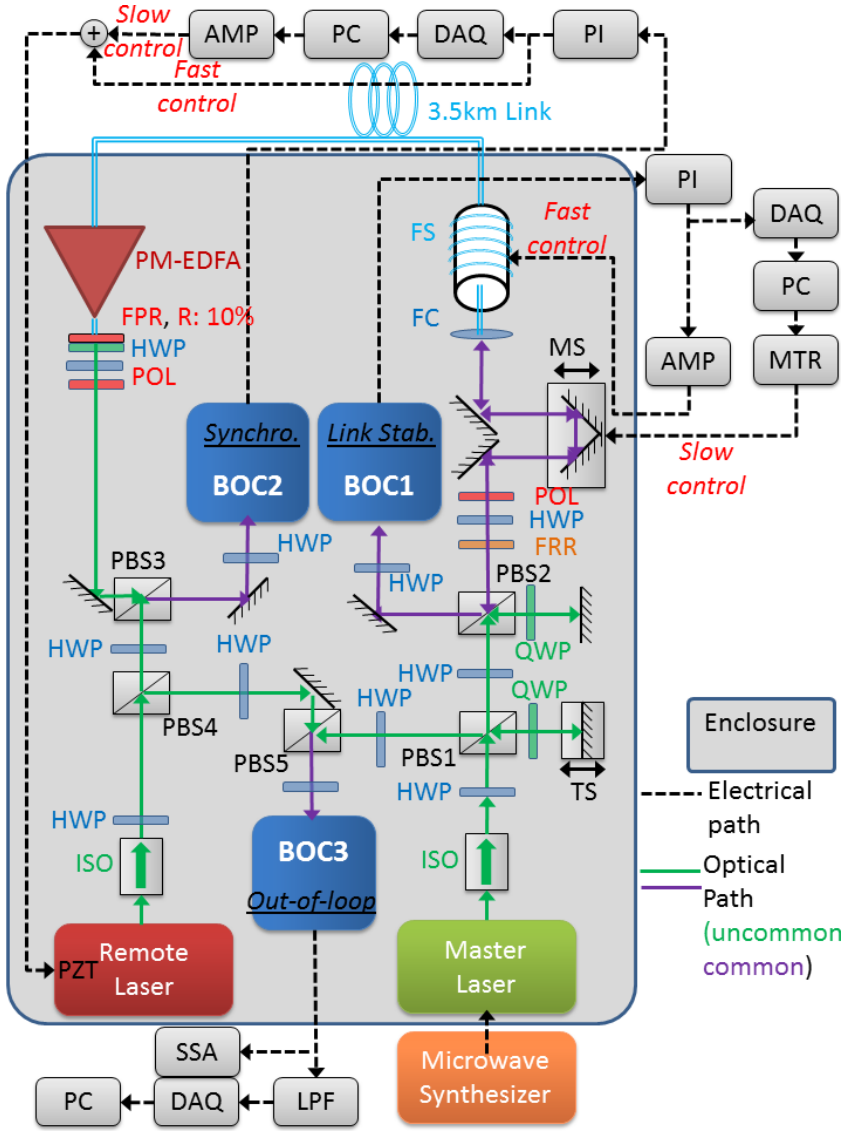

Fig. 5: Experimental setup for remote laser-to-laser synchronization. Abbreviations: ISO: isolator; HWP: half-wave plate; QWP: quarter-wave plate; PBS: polarization beam splitter; TS: translation stage; FRR: Faraday rotator; POL: polarizer; MS: motorized stage; FC: fiber coupler; FS: fiber stretcher; PMF: PM fiber; PMDCF: PM dispersion compensated fiber; PI: proportional-integral servo controller; SSA: signal source analyzer; LPF: low pass filter; DAQ: data acquisition card; AMP: amplifier; PC: computer; MTR: motor controller; PM-EDFA: PM Er doped fiber amplifier; FPR: fiber partial reflector; BOC: balanced optical cross-correlator.

demonstrate the preserved high-precision synchronization outcome.

The laser pair was the two Origami-15s described in Section II and BOC1 again stabilized the 3.5-km fiber link. Pulses from the link output and from the remote laser were combined at the beam splitter PBS3 and the timing mismatch between the two pulses was detected by BOC2. The BPD used here had a bandwidth of $100 \mathrm{MHz}$ at $50 \times 10^{3} \mathrm{~V} / \mathrm{A}$ transimpedance gain and the achieved timing sensitivity was $7.9 \mathrm{mV} / \mathrm{fs}$. The timing mismatch between the remote laser and the fiber link output pulse train was compensated by a feedback signal applied to its PZT. The out-of-loop performance of the remote synchronization system was evaluated by BOC 3 , whose timing sensitivity was $160 \mathrm{mV} / \mathrm{fs}$ measured with a BPD of $250 \mathrm{kHz}$ bandwidth at $10^{5} \mathrm{~V} / \mathrm{A}$ transimpedance gain.

\section{B. Results \& Discussion}

Fig. 6(a) shows the residual timing drift between the two remotely locked lasers measured by $\mathrm{BOC} 3$ with a $1-\mathrm{Hz} \mathrm{LPF}$. The result shows a RMS drift of only 0.88 fs and a maximum 

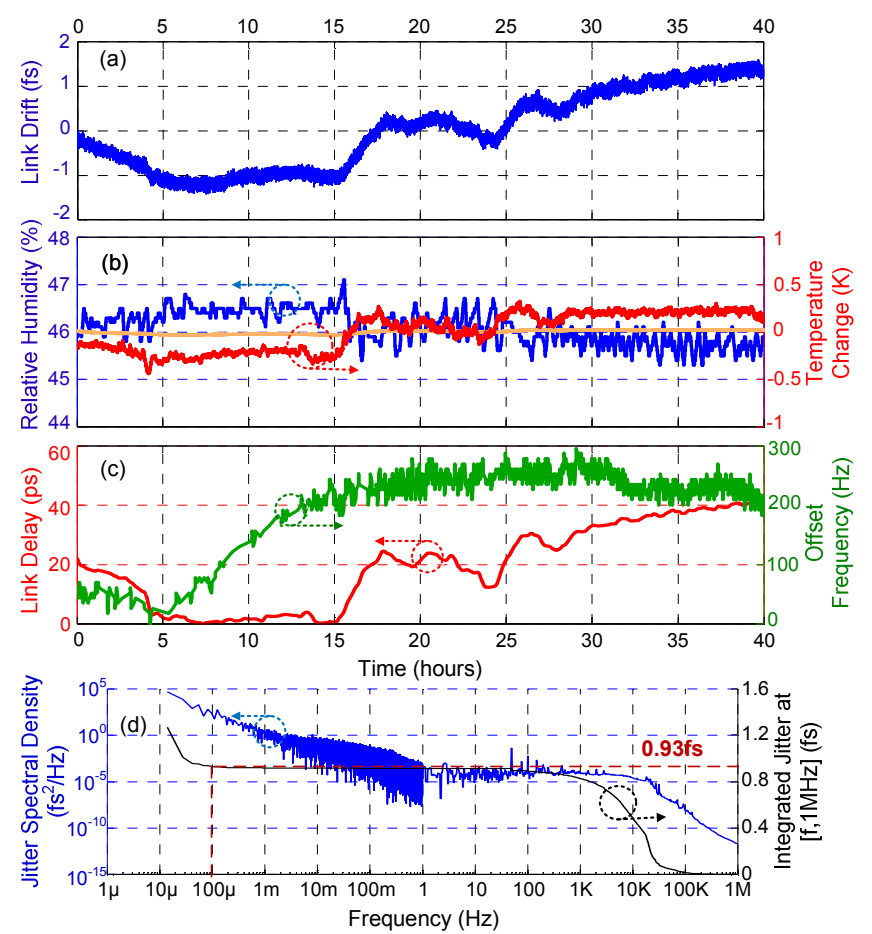

Fig. 6: Remote laser-to-laser synchronization results. (a) Timing drift over 40 hours of operation measured by BOC3; (b) environmental conditions during the measurement; blue curve shows the humidity change around the fiber link whereas red and orange curves show the temperatures on the case containing the fiber spool and on the breadboard respectively; (c) red curve: corrected link delay by the motorized stage, green curve: corrected offset frequency of the remote laser via its PZT; (d) blue curve: timing jitter spectral density between $11 \mu \mathrm{Hz}$ and $1 \mathrm{MHz}$, black curve: its integrated jitter.

deviation of about 3 fs over 40 hours of uninterrupted operation. As can be seen from Fig. 6(b), the link was exposed to harsher environmental conditions when compared with the conditions displayed in Fig. 4(b). Throughout the measurement, the temperature of the link changed by $0.7 \mathrm{~K}$ peak-to-peak and the humidity was fluctuating by $1.8 \%$. Furthermore, the resulting change in link length was more than $40 \mathrm{ps}$ and the remote lock corrected more than $300 \mathrm{~Hz}$ of frequency mismatch between the two lasers over 40 hours (red and green curves on Fig. 6(c) respectively). By taking the Fourier transform of the link drift data in Fig. 6(a) and then combining it with the high frequency jitter measurement results from the SSA, we obtained the complete jitter spectral density from $11 \mu \mathrm{Hz}$ to $1 \mathrm{MHz}$, as shown in Fig. 6(d). The integrated jitter was kept below 1 fs over 11 orders in the frequency range from $100 \mu \mathrm{Hz}$ to $1 \mathrm{MHz}$.

This measurement also revealed that there is a strong correlation between the observed out-of-loop drift and the delay applied by the motorized stage (mainly caused by the link temperature fluctuations). This could be the result of an unbalance in BOC1 caused by a misalignment of one of the beam paths, or an imperfect detector pair in the BPD. Current work is focused on the improvement of this balanced detection scheme and on the development of an integrated BOC using
PPKTP waveguides in order to eliminate alignment issues and increase the BOC timing sensitivity [8].

\section{CONCLUSION}

In summary, the pulsed-optical timing distribution scheme based on BOCs and a PM-fiber link is capable of achieving long-term stable, sub-fs precision timing distribution in realistic facility conditions. Jitter characterization of the OMO showed less 170 as integrated jitter for frequencies above 10 $\mathrm{kHz}$, confirming once more the excellent low-jitter properties of mode-locked lasers.

We have demonstrated timing stabilization of a $3.5-\mathrm{km}$ long fiber link under well controlled environmental conditions and achieved record-low 0.36 fs RMS link drift and jitter integrated from $100 \mu \mathrm{Hz}$ to $1 \mathrm{MHz}$. Finally, a remote modelocked laser was synchronized successfully to the OMO via the $3.5-\mathrm{km}$ link and the timing jitter between the two lasers was only 0.93 fs integrated from $100 \mu \mathrm{Hz}$ to $1 \mathrm{MHz}$.

Sub-fs precision for link-stabilization and remote laser locking over km distances has been reported before [6], [9]; however, this is the first demonstration of sub-fs precision for remote synchronization achieved under realistic facility conditions. We believe that the demonstrated timing link can be deployed in the European X-FEL to achieve femtosecond level timing distribution over the full length of the facility, helping to both monitor and control the electron bunch creation and hard X-ray pulse generation, to ultimately push the limits of spatial and temporal X-ray diffractive imaging.

\section{REFERENCES}

[1] M. Altarelli, R. Brinkmann, M. Chergui, W. Decking, B. Dobson, S Düsterer, G. Grübel, W. Graeff, H. Graafsma, and J. Hajdu, "XFEL: The European X-Ray Free-Electron Laser. Technical Design Report," DESY, 2006.

[2] J. Kim, J. A. Cox, J. Chen, and F. X. Kärtner, "Drift-free femtosecond timing svnchronization of remote optical and microwave sources," Nat. Photonics 2, 2008, pp. 733-736.

[3] T. R. Schibli, J. Kim, O. Kuzucu, J. T. Gopinath, S. N. Tandon, G. S. Petrich, L. A. Kolodziejski, J. G. Fujimoto, E. P. Ippen, and F. X. Kärtner, "Attosecond active synchronization of passively mode-locked lasers by balanced cross correlation,” Opt. Lett. 28, 2003, pp. 947-949.

[4] A. J. Benedick, J. G. Fujimoto, and F. X. Kärtner, "Optical flywheels with attosecond jitter," Nat. Photonics 6, 2012, pp. 97-100.

[5] J. A. Cox, A. H. Nejadmalayeri, J. Kim, and F. X. Kärtner, "Complete characterization of quantum-limited timing jitter in passively modelocked fiber lasers," Opt. Lett. 35, 2010, pp. 3522-3524.

[6] M. Xin, K. Safak, M. Y. Peng, P. T. Callahan and F. X. Kärtner, "Onefemtosecond, long-term stable remote laser synchronization over a 3.5km fiber link," submitted to Optics Express.

[7] J. A. Cox, "Sub-fs precision timing distribution, synchronization and coherent synthesis of ultrafast lasers," PhD Thesis, Massachusetts Institute of Technology, 2012.

[8] P. T. Callahan, K. Safak, P. Battle, T. D. Roberts and F. X. Kärtner, "Fiber-coupled balanced optical cross-correlator using PPKTP waveguides," submitted to Optics Express.

[9] M. Y. Peng, P.T. Callahan, A. H. Nejadmalayeri, S. Valente, M. Xin, L. Grüner-Nielsen, E. M. Monberg, M. Yan, J.M. Fini, and F. X. Kärtner, "Long-term stable, sub-femtosecond timing distribution via a $1.2-\mathrm{km}$ polarization-maintaining fiber link: approaching $10^{-21}$ link stability," Opt. Expr. 21, 2013, pp. 19982-19989. 